\title{
Non-Hermitian band topology from momentum-dependent relaxation in two-dimensional metals with spiral magnetism
}

\author{
Johannes Mitscherling (1) and Walter Metzner [ \\ Max Planck Institute for Solid State Research, D-70569 Stuttgart, Germany
}

(Received 27 July 2021; revised 14 October 2021; accepted 2 November 2021; published 15 November 2021)

\begin{abstract}
We study the emergence of non-Hermitian band topology in a two-dimensional metal with planar spiral magnetism due to a momentum-dependent relaxation rate. A sufficiently strong momentum dependence of the relaxation rate leads to exceptional points in the Brillouin zone, where the Hamiltonian is nondiagonalizable. The exceptional points appear in pairs with opposite topological charges and are connected by arc-shaped branch cuts. We show that exceptional points inside hole and electron pockets, which are generally present in a spiral magnetic state with a small magnetic gap, can cause a drastic change of the Fermi surface topology by merging those pockets at isolated points in the Brillouin zone. We derive simple rules for the evolution of the eigenstates under semiclassical motion through these crossing points, which yield geometric phases depending only on the Fermi surface topology. The spectral function observed in photoemission exhibits Fermi arcs. Its momentum dependence is smooth-despite of the nonanalyticities in the complex quasiparticle band structure.
\end{abstract}

DOI: 10.1103/PhysRevB.104.L201107

Introduction. The discovery of topological insulators [1,2] has triggered a systematic analysis and classification of topological features of band structures in solids. So far, the main focus has been on noninteracting electrons and superconductors in a mean-field picture, corresponding to Hermitian quadratic Hamiltonians [3]. Recently, there has been growing interest in topological features of non-Hermitian Hamiltonians [4]. In quantum many-body systems, these naturally arise in certain open systems, but also as effective Hamiltonians capturing relaxation processes in interacting and/or disordered closed systems [5-19]. A striking effect, which is unique to non-Hermitian systems, is the existence of exceptional points in momentum space where the Hamiltonian is not diagonalizable.

In this letter we show that the combination of two seemingly innocuous ingredients - spiral magnetic order in a two-dimensional metal and a momentum-dependent relaxation rate-can lead to a non-Hermitian Hamiltonian with nontrivial topological features, such as exceptional points and branch cuts in the Brillouin zone. Spiral order is a candidate for incommensurate magnetic order observed in cuprate superconductors [20-30], while relaxation rates with a pronounced momentum dependence arise naturally in two-dimensional systems with strong antiferromagnetic fluctuations [31-34]. We find that the momentum dependence of the relaxation rate can lead to a closing of the direct band gap between the quasiparticle bands $E_{\mathbf{p}}^{+}$and $E_{\mathbf{p}}^{-}$on

Published by the American Physical Society under the terms of the Creative Commons Attribution 4.0 International license. Further distribution of this work must maintain attribution to the author(s) and the published article's title, journal citation, and DOI. Open access publication funded by the Max Planck Society. one-dimensional branch cuts in the Brillouin zone, which terminate at exceptional points. These lines of degenerate quasiparticle bands, which are sometimes referred to as nonHermitian (bulk) Fermi arcs [4,5], are in general dispersive, in contrast to flat degenerate bands in some other systems $[5,16,35]$. In the dispersive case, hole and electron pockets merge at isolated momenta in the Brillouin zone where these degenerate bands cross the Fermi level, leading thus to a peculiar Fermi surface topology. Electrons traversing such crossing points along the Fermi surface acquire $\pi$-phase shifts, which can lead to a nontrivial geometric Berry phase. Surprisingly, we find that the nonanalyticity of the complex band at the exceptional points does not entail any singularity in the spectral function for single electron excitations. Nevertheless, the Fermi surface obtained from the spectral function seems truncated to Fermi arcs.

Spiral spin density waves. In a planar spiral spin density wave, the local magnetic moment has the form $\mathbf{S}_{i}=m \mathbf{n}_{i}$, where $m$ is a constant amplitude and $\mathbf{n}_{i}$ a site-dependent unit vector, which rotates in a fixed but arbitrary plane. For definiteness, we choose $\mathbf{n}_{i}$ to lie in the $x-y$ plane, such that $\mathbf{n}_{i}=\left(\cos \left(\mathbf{Q} \cdot \mathbf{R}_{i}\right), \sin \left(\mathbf{Q} \cdot \mathbf{R}_{i}\right), 0\right)$, where $\mathbf{Q}$ is the wave vector of the spin density wave. On a mean-field level, the planar spiral spin density wave is described by the two-band tightbinding Hamiltonian $H=\sum_{\mathbf{p}} \Psi_{\mathbf{p}}^{\dagger} H_{\mathbf{p}} \Psi_{\mathbf{p}}$, where the spinor $\Psi_{\mathbf{p}}=\left(c_{\mathbf{p}+\mathbf{Q}, \uparrow}, c_{\mathbf{p}, \downarrow}\right)$ collects the two spin components with a relative momentum shift $\mathbf{Q}$, and

$$
H_{\mathbf{p}}=\left(\begin{array}{cc}
\epsilon_{\mathbf{p}+\mathbf{Q}} & -\Delta \\
-\Delta & \epsilon_{\mathbf{p}}
\end{array}\right),
$$

where $\epsilon_{\mathbf{p}}$ is the (bare) band dispersion and $\Delta$ the magnetic gap [36]. The wave vector $\mathbf{Q}$ can be chosen arbitrarily. The simple two-band structure is due to a symmetry under combined lattice translations and spin rotations [37]. For $\mathbf{Q}=(0,0)$ and 
$\mathbf{Q}=(\pi, \pi)$ one recovers ferromagnetic and Néel antiferromagnetic order aligned in the $x y$ plane, respectively. In the following we consider incommensurate spiral order with wave vectors of the form $\mathbf{Q}=(\pi-2 \pi \eta, \pi)$ with $\eta>0$, as found in the hole-doped Hubbard and $t-J$ model [20-30]. Diagonalizing $H_{\mathbf{p}}$ one finds two quasiparticle bands $E_{\mathbf{p}}^{ \pm}$with a minimal direct gap $\Delta$, which generally results in a reconstructed Fermi surface with electron and/or hole pockets [28-30].

Non-Hermitian effective Hamiltonian. In interacting electron systems all the information on the Fermi surface, quasiparticle bands and decay rates, as well as the spectral function measured in photoemission, is encoded in the singleparticle Green's function. The bare Green's function of the noninteracting reference system is dressed by the self-energy, which receives contributions from the electron-electron interaction, and possibly from phonon and impurity scattering. In the low-frequency limit, the real part of the self-energy yields a renormalization of the band structure, and a reduction of the quasiparticle weight, while the imaginary part describes the quasiparticle relaxation rate $\Gamma_{\mathbf{p}}$. Here we discard the real part and focus on the more interesting effects of $\Gamma_{\mathbf{p}}$ in combination with spiral magnetic order. In the two-component spinor basis defined above, the retarded Green's function can then be written as

$$
\mathcal{G}_{\mathbf{p}}^{R}(\omega)=\left[\omega+\mu-\mathcal{H}_{\mathbf{p}}\right]^{-1},
$$

where the non-Hermitian Hamiltonian $\mathcal{H}_{\mathbf{p}}$ is defined as

$$
\mathcal{H}_{\mathbf{p}}=H_{\mathbf{p}}-i\left(\begin{array}{cc}
\Gamma_{\mathbf{p}+\mathbf{Q}} & 0 \\
0 & \Gamma_{\mathbf{p}}
\end{array}\right),
$$

with $H_{\mathbf{p}}$ from Eq. (1). Imaginary off-diagonal components have only minor consequences [38].

Exceptional points. $\mathcal{H}_{\mathbf{p}}$ has the complex eigenvalues

$$
\mathcal{E}_{\mathbf{p}}^{ \pm}=\epsilon_{\mathbf{p}}^{s} \pm \sqrt{\mathcal{D}_{\mathbf{p}}}-i \Gamma_{\mathbf{p}}^{s},
$$

with the discriminant

$$
\mathcal{D}_{\mathbf{p}}=\left(\epsilon_{\mathbf{p}}^{a}-i \Gamma_{\mathbf{p}}^{a}\right)^{2}+\Delta^{2},
$$

where $\epsilon_{\mathbf{p}}^{s / a}=\frac{1}{2}\left(\epsilon_{\mathbf{p}+\mathbf{Q}} \pm \epsilon_{\mathbf{p}}\right)$ and $\Gamma_{\mathbf{p}}^{s / a}=\frac{1}{2}\left(\Gamma_{\mathbf{p}+\mathbf{Q}} \pm \Gamma_{\mathbf{p}}\right)$ are symmetric and antisymmetric linear combinations. The condition $\mathcal{D}_{\mathbf{p}}=0$ defines the exceptional points, that is, the set of momenta at which $\mathcal{H}_{\mathbf{p}}$ is not diagonalizable. The momentum dependence of $\Gamma_{\mathbf{p}}$ is crucial for the existence of exceptional points. For $\Gamma_{\mathbf{p}}=\Gamma$ or $\Gamma_{\mathbf{p}}=\Gamma_{\mathbf{p}+\mathbf{Q}}$, we have $\Gamma_{\mathbf{p}}^{a}=0$, and thus $\mathcal{D}_{\mathbf{p}} \geqslant \Delta^{2}>0$ in the magnetically ordered phase. For $\Gamma_{\mathbf{p}}^{a} \neq 0$, the real and imaginary parts of $\mathcal{D}_{\mathbf{p}}$ yield two conditions for an exceptional point,

$$
\begin{aligned}
\epsilon_{\mathbf{p}} & =\epsilon_{\mathbf{p}+\mathbf{Q}}, \\
\left|\Gamma_{\mathbf{p}+\mathbf{Q}}-\Gamma_{\mathbf{p}}\right| & =2 \Delta,
\end{aligned}
$$

which need to be satisfied simultaneously. The second condition requires a relaxation rate that exceeds the magnetic gap at particular momenta. The exceptional points can be classified by a topological charge $v_{i}= \pm \frac{1}{2}$ via

$$
v_{i}=-\frac{1}{2 \pi} \oint_{\Gamma_{i}} d \mathbf{p} \cdot \nabla_{\mathbf{p}} \arg \left[\mathcal{E}_{\mathbf{p}}^{+}-\mathcal{E}_{\mathbf{p}}^{-}\right]
$$

where $\Gamma_{i}$ is a closed contour encircling the $i$ th exceptional point counterclockwise $[5,39,40]$. Exceptional points with opposite charge are connected by branch cuts where $\mathcal{D}_{\mathbf{p}}$ is real and negative.

Fermi surface reconstruction. As an example, we assume a tight-binding dispersion on a square lattice, a magnetic gap $\Delta$ and a wave vector $\mathbf{Q}=(\pi-2 \pi \eta, \pi)$ such that several hole and electron pockets are present, confined by momenta at which the lower and upper quasiparticle bands cross the Fermi level, respectively. The dispersion has the form

$$
\begin{aligned}
\epsilon_{\mathbf{p}}= & -2 t\left(\cos p_{x}+\cos p_{y}\right)-4 t^{\prime} \cos p_{x} \cos p_{y} \\
& -2 t^{\prime \prime}\left[\cos \left(2 p_{x}\right)+\cos \left(2 p_{y}\right)\right],
\end{aligned}
$$

where $t, t^{\prime}$, and $t^{\prime \prime}$ are hopping amplitudes between nearest, next-nearest, and third-nearest neighbors, respectively. We use $t$ as our energy unit, and we choose $t^{\prime} / t=-0.17$ and $t^{\prime \prime} / t=0.05$, as widely used for $\mathrm{La}_{2-x} \mathrm{Sr}_{x} \mathrm{CuO}_{4}$ (LSCO) superconductors $[29,30,41]$. The parameters of the magnetic order $\Delta / t=0.144$ and $\eta=0.106$ are taken from recent DMFT results for the two-dimensional Hubbard model with LSCO parameters at a hole doping $p=1-n=0.177$ [30].

For the momentum-dependent relaxation rate $\Gamma_{\mathbf{p}}$ we assume a $d$-wave form

$$
\Gamma_{\mathbf{p}}=\gamma_{0}+\frac{\gamma_{d}}{4}\left(\cos p_{x}-\cos p_{y}\right)^{2},
$$

with $\gamma_{0}, \gamma_{d} \geqslant 0$, which has its minimal value $\left(\Gamma_{\min }=\gamma_{0}\right)$ along the Brillouin zone diagonals, and its maximal value $\left(\Gamma_{\max }=\gamma_{0}+\gamma_{d}\right)$ at the points $(\pi, 0)$ and $(0, \pi)$ on the $p_{x}$ and $p_{y}$ axis. The relaxation rate in a cuprate compound from the LSCO family has recently been determined experimentally via angle-resolved magneto-resistance measurements in the overdoped regime at various temperatures [42], yielding an estimate $\gamma_{0} / t \approx 0.015$ and $\gamma_{d} / t \approx 0.15$. For a given relaxation rate $\Gamma_{\mathbf{p}}$, the condition (7) can always be satisfied for a sufficiently small gap, for instance, near the onset of magnetic order at a quantum critical point. For a better visualization of the topological effects, we choose a sizable magnetic gap and consider relatively large values for the relaxation rate, namely $\gamma_{0} / t=0.05$, and $\gamma_{d} / t \leqslant 1.6$. We fix the doping level at $p=0.177$. All results are obtained at zero temperature.

In Fig. 1 (top row) we show the "nesting" lines defined by Eq. (6), and the lines corresponding to the condition (7) for different $\gamma_{d} / t$. Exceptional points where these lines cross exist for $\gamma_{d} / t \geqslant 1.0$. Changing parameters, exceptional points can be created or annihilated only in pairs with opposite topological charge. In the bottom row of Fig. 1 we show the quasiparticle Fermi surfaces. Electron and hole pockets are disconnected for $\gamma_{d} / t=0$ and 0.8 , while for larger $\gamma_{d} / t$ they merge at isolated momenta on the branch cuts.

In Fig. 2 we show the real and imaginary parts of the quasiparticle bands $\mathcal{E}_{\mathbf{p}}^{ \pm}$for $\gamma_{d} / t=1$ as a function of $p_{x}$ along the upper nesting line in Fig. 1, where the discriminant $\mathcal{D}_{\mathbf{p}}$ is real (since $\epsilon_{\mathbf{p}}^{a}=0$ ). For $\left|\Gamma_{\mathbf{p}+\mathbf{Q}}-\Gamma_{\mathbf{p}}\right|<2 \Delta$, the square root in Eq. (4) is real, such that $E_{\mathbf{p}}^{ \pm}=\operatorname{Re} \mathcal{E}_{\mathbf{p}}^{ \pm}$describes two separate bands. For $\left|\Gamma_{\mathbf{p}+\mathbf{Q}}-\Gamma_{\mathbf{p}}\right|>2 \Delta$, that is, on the branch cut, the square root in Eq. (4) is purely imaginary such that $E_{\mathbf{p}}^{+}=E_{\mathbf{p}}^{-}$, while now $\Gamma_{\mathbf{p}}^{ \pm}=-\operatorname{Im} \mathcal{E}_{\mathbf{p}}^{ \pm}$assumes two distinct values. At the exceptional points, where $\left|\Gamma_{\mathbf{p}+\mathbf{Q}}-\Gamma_{\mathbf{p}}\right|=2 \Delta$, both real and imaginary parts of the two complex bands $\mathcal{E}_{\mathbf{p}}^{ \pm}$collapse to a 

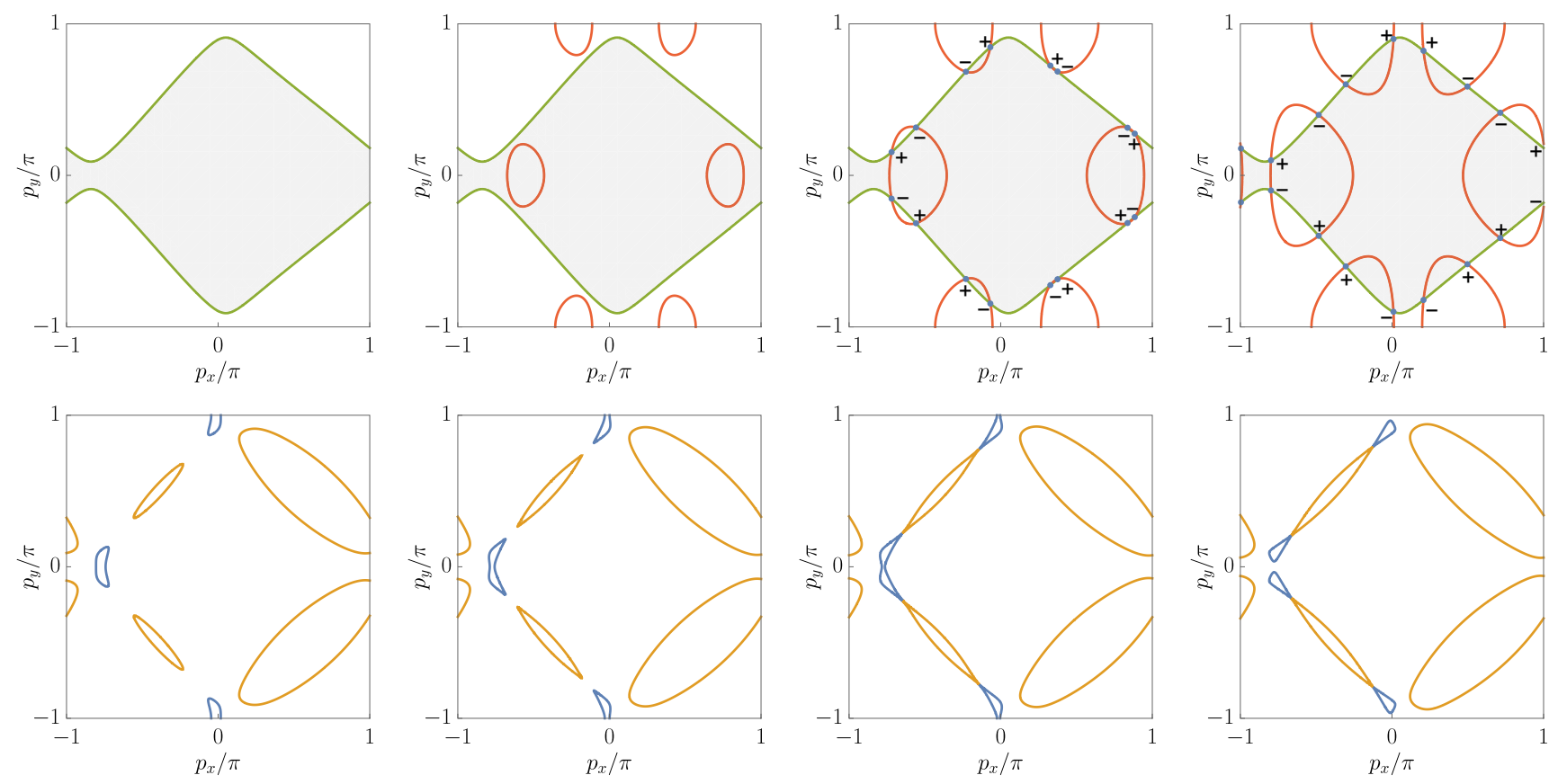

FIG. 1. From left to right: $\gamma_{d} / t=0,0.8,1.0$, and 1.6. Upper row: The conditions given by Eq. (6) (green "nesting" lines), which separates the region of $\epsilon_{\mathbf{p}}^{a}>0$ (gray) and $\epsilon_{\mathbf{p}}^{a}<0$ (white), and Eq. (7) (red lines). Exceptional points are situated at the intersection of both lines, and carry the topological charge $v_{i}= \pm \frac{1}{2}$. Lower row: The quasiparticle Fermi surfaces defined by $\operatorname{Re} \mathcal{E}_{\mathbf{p}}^{ \pm}=\mu$ for fixed doping $p=0.177$. Hole pockets (orange) and electron pockets (blue) merge at isolated momenta on the branch cuts, that is, on the parts of the nesting line between exceptional points of opposite charge.

single value. The degenerate band $E_{\mathbf{p}}^{+}=E_{\mathbf{p}}^{-}$on the branch cut is dispersive. Thus, it intersects the Fermi level only at isolated momenta, which leads to the peculiar Fermi surface topology in Fig. 1. The merging of hole and electron pockets at single isolated momenta is a generic consequence of exceptional points with opposite topological charge inside the pockets and, thus, not restricted to our specific realization by the particular form of the dispersion in Eq. (9) or the relaxation rate in Eq. (10).

Semiclassical transport through crossing points. In a semiclassical description of the electron dynamics, the momentum of electrons changes smoothly in the direction of the applied force [43]. The Lorentz force acts perpendicularly to the electron velocity, such that a magnetic field makes low-energy electrons move along the Fermi surface. We now clarify how electrons move semiclassically through the crossing points. There are potentially six paths on the Fermi surface. (see Fig. 3). We study the evolution of a biorthonormal basis with left and right eigenstates $\left|L_{\mathbf{p}}^{n}\right\rangle$ and $\left|R_{\mathbf{p}}^{n}\right\rangle$ for the bands $n= \pm$ when $\mathbf{p}$ traverses the crossing point $[44,45]$. Since the Hamiltonian in Eq. (3) is symmetric, $\mathcal{H}_{\mathbf{p}}=\mathcal{H}_{\mathbf{p}}^{\text {tr }}$, we can choose a gauge such that $\left|L_{\mathbf{p}}^{n}\right\rangle=\left(\left|R_{\mathbf{p}}^{n}\right\rangle\right)^{*}$. Thus, the Berry connection $i\left\langle L_{\mathbf{p}}^{ \pm} \mid \partial_{p_{\alpha}} R_{\mathbf{p}}^{ \pm}\right\rangle$vanishes and the geometric phase $\gamma_{B}$ is determined exclusively by the overlap of the initial and final states [46]. For definiteness, the remaining gauge freedom $\left|R_{\mathbf{p}}^{n}\right\rangle \rightarrow \pm\left|R_{\mathbf{p}}^{n}\right\rangle$ has also been fixed.

In the Supplemental Material [38] we show that only the two diagonal paths allow for a continuous evolution of the eigenstates through the crossing point. The phase shift is determined by the sign change of $\epsilon_{\mathbf{p}}^{a}$. With the shorthand notation
$\left|n_{\mathbf{p}}\right\rangle=\left|R_{\mathbf{p}}^{n}\right\rangle$, the transition of states at the crossing point is given by

$$
\left|+_{\mathbf{p}}\right\rangle \rightleftharpoons-\left|-_{\mathbf{p}}\right\rangle \quad \text { and } \quad\left|-_{\mathbf{p}}\right\rangle \rightleftharpoons\left|{ }_{\mathbf{p}}\right\rangle,
$$

where the transition from left to right is when crossing with sign change $+\rightarrow-$ (gray to white in Fig. 3) and the evolution from right to left is when crossing with sign change $-\rightarrow+$ (white to gray in Fig. 3). The first rule in Eq. (11) involves a minus sign beside the well-known swapping of the eigenstates [4]. The velocities $\partial_{p_{\alpha}} E_{\mathbf{p}}^{ \pm}$are smooth and finite at the crossing point [38]. Note that the rules in Eq. (11) are gauge dependent, but the total geometric phase accumulated in a closed loop is a gauge independent quantity.
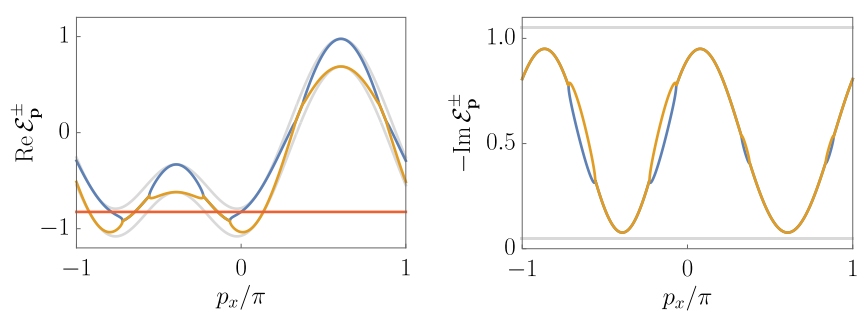

FIG. 2. Real part (left) and imaginary part (right) of the complex quasiparticle bands $\mathcal{E}_{\mathbf{p}}^{ \pm}$for $\gamma_{d} / t=1$ on the upper nesting line where $\epsilon_{\mathbf{p}}=\epsilon_{\mathbf{p}+\mathbf{Q}}$ (see upper row in Fig. 1) as a function of $p_{x}$. Left: The band gap closes between the exceptional points. The bands for $\gamma_{d}=$ 0 are shown for comparison (gray lines). The chemical potential $\mu$ is indicated by the red line. Right: The quasiparticle relaxation rate $\Gamma_{\mathbf{p}}^{ \pm}=-\operatorname{Im} \mathcal{E}_{\mathbf{p}}^{ \pm}$is double-valued between the exceptional points. The gray lines indicate $\Gamma_{\min }$ and $\Gamma_{\max }$. Note that $\Gamma_{\mathbf{p}}^{ \pm}$is always positive. 


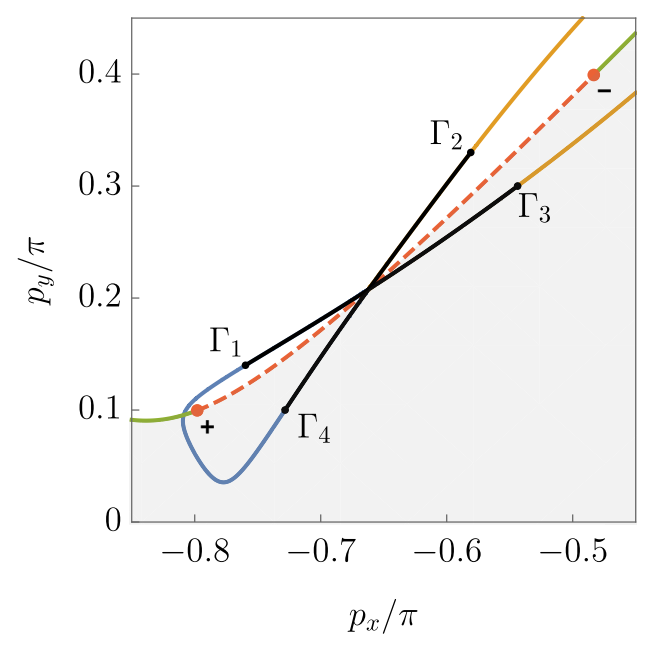

FIG. 3. Close-up of a crossing point for $\gamma_{d} / t=1.6$. The electron (blue) and hole (orange) pockets encircle exceptional points (red dots) of opposite charge. They merge at one point on the branch cut (red-dashed line). Only the two diagonal paths from $\Gamma_{1}$ to $\Gamma_{3}$ and from $\Gamma_{2}$ to $\Gamma_{4}$ are continuously connected. The crossing rules depend on the sign change between the regions $\epsilon_{\mathbf{p}}^{a}>0$ (gray) and $\epsilon_{\mathbf{p}}^{a}<0$ (white).

In Fig. 4 we sketch the evolution of the eigenstates for electrons moving along the Fermi surface according to Eq. (11). For the pockets in Fig. 1 we find a vanishing geometric phase $\gamma_{B}=0$ after a completed round, but a relative phase difference $\pi$ on opposite sides of the hole pocket [step ii) and iv)]. We predict a geometric phase $\gamma_{B}=\pi$ for an "eight" topology of a merged electron and hole pocket, so that the original state is then recovered only after two rounds.

Quantum oscillation experiments at sufficiently large magnetic fields $\omega_{c} \tau>1$, where $\omega_{c}$ is the cyclotron frequency and $\tau=1 / 2 \Gamma$, can be used to measure the Fermi surface topology. Thus, the merging of electron and hole pockets (see Fig. 1) is visible at least in principle in the spectrum of quantum oscillations. A geometric phase can be observed experimentally as a phase shift in quantum oscillations [47]. A detailed microscopic or semiclassical analysis of transport in non-Hermitian systems is still ongoing research [29,48-56] and beyond the scope of this paper.
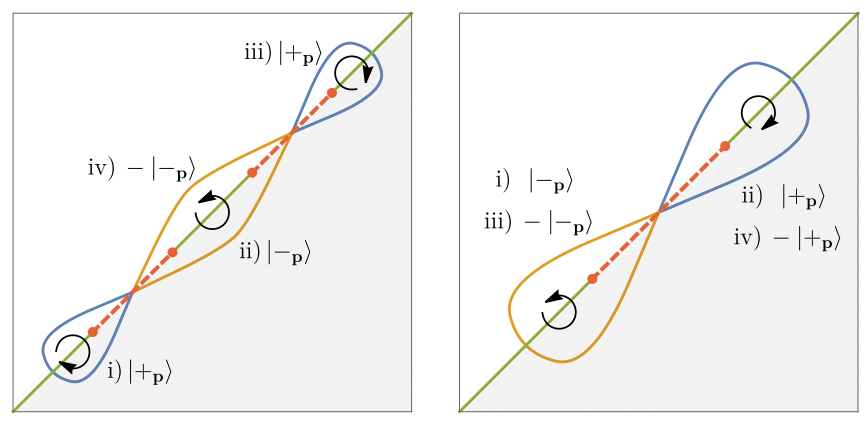

FIG. 4. Evolution of the eigenstates i) - iv) for electrons moving along the Fermi surface in the arrow directions. Left: The "doubleeight" topology appearing in Fig. 1. Right: A hypothetical "eight" topology.
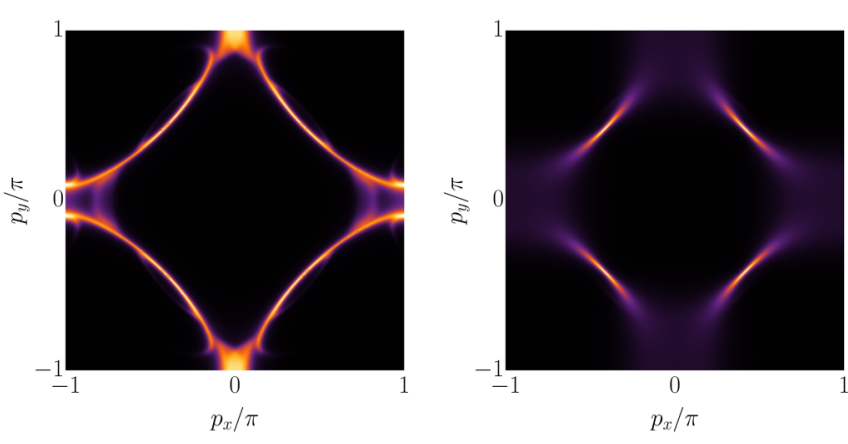

FIG. 5. The spectral function for single-particle excitations $A_{\mathbf{p}}(0)$ for $\gamma_{d} / t=0$ (left) and $\gamma_{d} / t=1$ (right).

Spectral functions. The quasiparticle spectral function is given by the diagonal matrix $\tilde{\mathcal{A}}_{\mathbf{p}}(\omega)=-\frac{1}{\pi} \operatorname{Im} \tilde{\mathcal{G}}_{\mathbf{p}}^{R}(\omega)$, where $\tilde{\mathcal{G}}_{\mathbf{p}}^{R}(\omega)=\left[\omega+\mu-\tilde{\mathcal{H}}_{\mathbf{p}}\right]^{-1}$ is the retarded Green's function in the quasiparticle basis, and $\tilde{\mathcal{H}}_{\mathbf{p}}$ is the diagonalized nonHermitian Hamiltonian with the eigenvalues $\mathcal{E}_{\mathbf{p}}^{ \pm}$from Eq. (4). The quasiparticle spectral functions are thus Lorentzians with positions $\epsilon_{\mathbf{p}}^{s} \pm \epsilon_{\mathbf{p}}^{\mathcal{D}}-\mu$ and widths $\Gamma_{\mathbf{p}}^{s} \mp \Gamma_{\mathbf{p}}^{\mathcal{D}}$, where $\epsilon_{\mathbf{p}}^{\mathcal{D}}=$ $\operatorname{Re} \sqrt{\mathcal{D}_{\mathbf{p}}}$ and $\Gamma_{\mathbf{p}}^{\mathcal{D}}=\operatorname{Im} \sqrt{\mathcal{D}_{\mathbf{p}}}$.

The spectral function matrix in the bare band basis is given by $\mathcal{A}_{\mathbf{p}}(\omega)=\frac{1}{2 \pi i}\left(\mathcal{G}_{\mathbf{p}}^{A}(\omega)-\mathcal{G}_{\mathbf{p}}^{R}(\omega)\right)$ with $\mathcal{G}_{\mathbf{p}}^{A}(\omega)=\left[\mathcal{G}_{\mathbf{p}}^{R}(\omega)\right]^{\dagger}$ The Green's function in the bare band basis is related to the quasiparticle Green's function by $\mathcal{G}_{\mathbf{p}}^{R}(\omega)=\mathcal{U}_{\mathbf{p}} \tilde{\mathcal{G}}_{\mathbf{p}}^{R}(\omega) \mathcal{U}_{\mathbf{p}}^{-1}$, where the matrix $\mathcal{U}_{\mathbf{p}}$ diagonalizes $\mathcal{H}_{\mathbf{p}}$ for all momenta except, of course, the exceptional ones. The diagonal elements of $\mathcal{A}_{\mathbf{p}}(\omega)$ are obtained as [38]

$$
\begin{aligned}
A_{\mathbf{p}}^{\uparrow / \downarrow}(\omega)= & \frac{1}{2}\left[\tilde{A}_{\mathbf{p}}^{+}(\omega)+\tilde{A}_{\mathbf{p}}^{-}(\omega)\right], \\
& \pm\left(\frac{1}{2} \frac{\epsilon_{\mathbf{p}}^{a} \epsilon_{\mathbf{p}}^{\mathcal{D}}-\Gamma_{\mathbf{p}}^{a} \Gamma_{\mathbf{p}}^{\mathcal{D}}}{\left(\epsilon_{\mathbf{p}}^{\mathcal{D}}\right)^{2}+\left(\Gamma_{\mathbf{p}}^{\mathcal{D}}\right)^{2}}\left[\tilde{A}_{\mathbf{p}}^{+}(\omega)-\tilde{A}_{\mathbf{p}}^{-}(\omega)\right],\right. \\
& \left.+\frac{1}{2 \pi} \frac{\epsilon_{\mathbf{p}}^{a} \Gamma_{\mathbf{p}}^{\mathcal{D}}+\Gamma_{\mathbf{p}}^{a} \epsilon_{\mathbf{p}}^{\mathcal{D}}}{\left(\epsilon_{\mathbf{p}}^{\mathcal{D}}\right)^{2}+\left(\Gamma_{\mathbf{p}}^{\mathcal{D}}\right)^{2}}\left[\tilde{P}_{\mathbf{p}}^{+}(\omega)-\tilde{P}_{\mathbf{p}}^{-}(\omega)\right]\right),
\end{aligned}
$$

where $\tilde{P}_{\mathbf{p}}^{ \pm}(\omega)$ are the elements of the diagonal matrix $\tilde{\mathcal{P}}_{\mathbf{p}}(\omega)=$ $\operatorname{Re} \tilde{\mathcal{G}}_{\mathbf{p}}^{R}(\omega)$. Due to the momentum shift $\mathbf{Q}$ in the spinor $\Psi_{\mathbf{p}}$, the total spectral function for the physical single electron excitations reads

$$
A_{\mathbf{p}}(\omega)=A_{\mathbf{p}-\mathbf{Q}}^{\uparrow}(\omega)+A_{\mathbf{p}}^{\downarrow}(\omega) .
$$

For $\Gamma_{\mathbf{p}}^{a}=0$ we have $\Gamma_{\mathbf{p}}^{\mathcal{D}}=0$, so that we recover the wellknown result for a momentum-independent relaxation rate $[28,38]$. The appearance of the term (14) is directly linked to a nonzero $\Gamma_{\mathbf{p}}^{a}$. In the Supplemental Material [38] we analyze the effect of exceptional points. Both the second term in (13) and the third term in (14) are discontinuous at the exceptional points. Due to the phase shift $\frac{\pi}{2}$ in $\sqrt{\mathcal{D}_{\mathbf{p}}}$ when crossing the exceptional point, the two contributions are mapped onto each other. Thus, the sum of both is continuous. In other words, the nonanalyticity of the complex band at the exceptional points does not appear in $A_{\mathbf{p}}(\omega)$.

In Fig. 5 we show the spectral function $A_{\mathbf{p}}(\omega)$ at $\omega=0$ for $\gamma_{d} / t=0$ and $\gamma_{d} / t=1$. The spectral weight is strongly 
suppressed for momenta away from the bare Fermi surface [28,38]. Moreover, the angle dependence of $\Gamma_{\mathbf{p}}$ reduces the spectral weight in the antinodal region, such that only Fermi arcs in the nodal region are visible.

Conclusions. We have analyzed the non-Hermitian band topology resulting from a momentum-dependent relaxation rate $\Gamma_{\mathbf{p}}$ in a two-dimensional metal with spiral magnetic order. We provided a concrete example for a specific band dispersion and relaxation rate. We find that arc-shaped branch cuts connecting exceptional points with opposite topological charges appear in the Brillouin zone. Exceptional points inside hole and electron pockets lead to a peculiar Fermi surface topology with pockets merging at isolated points in the Brillouin zone. We have derived rules for the evolution of eigenstates under semiclassical motion through these crossing points, from which geometric phases associated with the Fermi surface topology can be obtained. The change of the Fermi sur- face topology and the geometric phase are visible at least in principle via quantum oscillations. The spectral function for single-particle excitations, which can be observed in photoemission experiments, exhibits Fermi arcs. Its momentum dependence is however smooth, due to subtle cancellations of the nonanalyticities in the complex quasiparticle band structure.

Our paper provides an example for an intriguing nonHermitian topological band structure emerging from a combination of conventional ingredients, in an electron system that was hitherto expected to be topologically trivial. Following this paradigm, we expect the discovery of other condensed matter systems with an interesting non-Hermitian band topology.

Acknowledgments. We are very grateful to P. M. Bonetti, G. Grissonnanche, S. Groenendijk, M. M. Hirschmann, and especially A. Schnyder for valuable discussions.
[1] M. Z. Hasan and C. L. Kane, Colloqium: Topological insulators, Rev. Mod. Phys. 82, 3045 (2010).

[2] J. E. Moore, The birth of topological insulators, Nature (London) 464, 194 (2010).

[3] C.-K. Chiu, J. C. Y. Teo, A. P. Schnyder, and S. Ryu, Classification of topological quantum matter with symmetries, Rev. Mod. Phys. 88, 035005 (2016).

[4] For a recent overview, see E. J. Bergholtz, J. C. Budich, and F. K. Kunst, Exceptional topology of non-Hermitian systems, Rev. Mod. Phys. 93, 015005 (2021), and references therein.

[5] V. Kozii and L. Fu, Non-Hermitian topological theory of finitelifetime quasiparticles: Prediction of bulk Fermi arc due to exceptional point, arXiv:1708.05841.

[6] T. Yoshida, R. Peters, and N. Kawakami, Non-Hermitian perspective of the band structure in heavy-fermion systems, Phys. Rev. B 98, 035141 (2018).

[7] A. A. Zyuzin and A. Y. Zyuzin, Flat band in disorder-driven non-Hermitian Weyl semimetals, Phys. Rev. B 97, 041203(R) (2018).

[8] K. Kimura, T. Yoshida, and N. Kawakami, Fermion Doubling Chiral-symmetry protected exceptional torus in correlated nodal-line semimetals, Phys. Rev. B 100, 115124 (2019).

[9] P. A. McClarty and J. G. Rau, Non-Hermitian topology of spontaneous magnon decay, Phys. Rev. B 100, 100405(R) (2019).

[10] K. Moors, A. A. Zyuzin, A. Y. Zyuzin, R. P. Tiwari, and T. L. Schmidt, Disorder-driven exceptional lines and Femri ribbons in tilted nodal-line semimetals, Phys. Rev. B 99, 041116(R) (2019).

[11] M. Papaj, H. Isobe, and L. Fu, Nodal arc of disordered Dirac fermions and non-Hermitian band theory, Phys. Rev. B 99, 201107(R) (2019).

[12] T. Yoshida, R. Peters, N. Kawakami, and Y. Hatsugai, Symmetry-protected exceptional rings in two-dimensional correlated systems with chiral symmetry, Phys. Rev. B 99, 121101(R) (2019).

[13] A. A. Zyuzin and P. Simon, Disorder-induced exceptional points and nodal lines in Dirac superconductors, Phys. Rev. B 99, 165145 (2019).

[14] R. Aquino and D. G. Barci, Exceptional points in Fermi liquids with quadrupolar interactions, Phys. Rev. B 102, 201110(R) (2020).
[15] Y. Michishita, T. Yoshida, and R. Peters, Relationship between exceptional points and the Kondo effect in $f$-electron materials, Phys. Rev. B 101, 085122 (2020).

[16] Y. Nagai, Y. Qi, H. Isobe, V. Kozii, and L. Fu, DMFT Reveals the Non-Hermitian Topology and Fermi Arcs in HeavyFermion Systems, Phys. Rev. Lett. 125, 227204 (2020).

[17] L. Crippa, J. C. Budich, and G. Sangiovanni, Fourth-order exceptional points in correlated quantum many-body systems, Phys. Rev. B 104, 121109 (2021).

[18] B. Michen, T. Micallo, and J. C. Budich, Exceptional nonHermitian phases in disordered quantum wires, Phys. Rev. B 104, 035413 (2021).

[19] R. Rausch, R. Peters, and T. Yoshida, Exceptional points in the one-dimensional Hubbard model, New J. Phys. 23, 013011 (2021).

[20] B. I. Shraiman and E. D. Siggia, Spiral Phase of a Doped Quantum Antiferromagnet, Phys. Rev. Lett. 62, 1564 (1989).

[21] K. Machida, Magnetism in $\mathrm{La}_{2} \mathrm{CuO}_{4}$ based compounds, Physica C 158, 192 (1989).

[22] T. Dombre, Modulated spiral phases in doped quantum antiferromagnets, J. Phys. (France) I 51, 847 (1990).

[23] R. Fresard, M. Dzierzawa, and P. Wölfle, Slave-Boson approach to spiral magnetic order in the Hubbard model, Europhys. Lett. 15, 325 (1991).

[24] A. V. Chubukov and D. M. Frenkel, Renormalized perturbation theory of magnetic instabilities in the two-dimensional Hubbard model at small doping, Phys. Rev. B 46, 11884 (1992).

[25] A. V. Chubukov and K. A. Musaelian, Magnetic phases of the two-dimensional Hubbard model at low doping, Phys. Rev. B 51, 12605 (1995).

[26] V. N. Kotov and O. P. Sushkov, Stability of the spiral phase in the two-dimensional extended $t-J$ model, Phys. Rev. B 70, 195105 (2004).

[27] H. Yamase, A. Eberlein, and W. Metzner, Coexistence of Incommensurate Magnetism and Superconductivity in the TwoDimensional Hubbard Model, Phys. Rev. Lett. 116, 096402 (2016).

[28] A. Eberlein, W. Metzner, S. Sachdev, and H. Yamase, Fermi Surface Reconstruction and Drop in the Hall Number Due to Spiral Antiferromagnetism in High- $T_{c}$ Cuprates, Phys. Rev. Lett. 117, 187001 (2016). 
[29] J. Mitscherling and W. Metzner, Longitudinal conductivity and Hall coefficient in two-dimensional metals with spiral magnetic order, Phys. Rev. B 98, 195126 (2018).

[30] P. M. Bonetti, J. Mitscherling, D. Vilardi, and W. Metzner, Charge carrier drop at the onset of pseudogap behavior in the two-dimensional Hubbard model, Phys. Rev. B 101, 165142 (2020).

[31] A. P. Kampf and J. R. Schrieffer, Pseudogaps and the spin-bag approach to high- $T_{c}$ superconductivity, Phys. Rev. B 41, 6399 (1990); Spectral function and photoemission spectra in antiferromagnetically correlated metals, 42, 7967 (1990).

[32] J. Vilk and A.-M. Tremblay, Non-perturbative many-body approach to the Hubbard model and single-particle pseudogap, J. Phys. (France) 7, 1309 (1997).

[33] A. A. Katanin and A. Kampf, Quasiparticle Anisotropy and Pseudogap Formation from a Weak-Coupling Renormalization Point of View, Phys. Rev. Lett. 93, 106406 (2004).

[34] D. Rohe and W. Metzner, Pseudogap at hot spots in the twodimensional Hubbard model at weak coupling, Phys. Rev. B 71, 115116 (2005).

[35] H. Zhou, C. Peng, Y. Yoon, C. W. Hsu, K. A. Nelson, L. Fu, J. D. Joannopoulos, M. Soljačić, and B. Zhen, Observation of bulk Fermi arc and polarization half charge from paired exceptional points, Science 359, 1009 (2018).

[36] P. A. Igoshev, M. A. Timirgazin, A. A. Katanin, A. K. Arzhnikov, and V. Yu. Irkhin, Incommensurate magnetic order and phase separation in the two-dimensional Hubbard model with nearest- and next-nearest-neighbor hopping, Phys. Rev. B 81, 094407 (2010).

[37] L. M. Sandratskii, Noncollinear magnetism in itinerant-electron systems: Theory and applications, Adv. Phys. 47, 91 (1998).

[38] See Supplemental Material at http://link.aps.org/supplemental/ 10.1103/PhysRevB.104.L201107 for further details on (i) the special case of a non-Hermitian part proportional to the identity matrix, (ii) the consequences of off-diagonal self-energy components, (iii) the behavior of the quasiparticle bands and eigenstates when crossing the branch cut, and (iv) the derivation and properties of the spectral functions.

[39] H. Shen, B. Zhen, and L. Fu, Topological Band Theory for NonHermitian Hamiltonians, Phys. Rev. Lett. 120, 146402 (2018).

[40] Z. Yang, A. P. Schnyder, J. Hu, and C.-K. Chiu, Fermion Doubling Theorems in Two-Dimensional Non-Hermitian Systems for Fermi Points and Exceptional Points, Phys. Rev. Lett. 126, 086401 (2021).

[41] S. Verret, O. Simard, M. Charlebois, D. Sénéchal, and A.-M. S. Tremblay, Phenomenological theories of the low-temperature pseudogap: Hall number, specific heat, and Seebeck coefficient, Phys. Rev. B 96, 125139 (2017).

[42] Y. Fang, G. Grissonananche, A. Legros, S. Verret, F. Laliberté, C. Collignon, A. Ataei, M. Dion, J. Zhou, D. Graf, M. J. Lawler, P. Goddard, L. Taillefer, and B. J. Ramshaw, Fermi surface transformation at the pseudogap critical point of a cuprate superconductor, arXiv:2004.01725.

[43] N. W. Ashcroft and N. D. Mermin, Solid State Physics (Saunders College, Philadelphia, 1976).

[44] D. C. Brody, Biorthogonal quantum mechanics, J. Phys. A: Math. Theor. 47, 035305 (2014).

[45] The right eigenstates are defined by the eigenvalue equation $\mathcal{H}_{\mathbf{p}}\left|R_{\mathbf{p}}^{n}\right\rangle=\mathcal{E}_{\mathbf{p}}^{n}\left|R_{\mathbf{p}}^{n}\right\rangle$. The left eigenstates are defined by the eigenvalue equation $\mathcal{H}_{\mathbf{p}}^{\dagger}\left|L_{\mathbf{p}}^{n}\right\rangle=\left(\mathcal{E}_{\mathbf{p}}^{n}\right)^{*}\left|L_{\mathbf{p}}^{n}\right\rangle$. Orthonormality is defined by $\left\langle L_{\mathbf{p}}^{n} \mid R_{\mathbf{p}}^{n}\right\rangle=\delta_{n m}$, where $\left\langle L_{\mathbf{p}}^{n}\right|=\left(\left|L_{\mathbf{p}}^{n}\right\rangle\right)^{\dagger}$. Further properties are discussed in the Supplemental Material [38].

[46] F. Keck, H. J. Korsch, and S. Mossmann, Unfolding a diabolic point: A generalized crossing scenario, J. Phys. A: Math. Gen. 36, 2125 (2003).

[47] D. Shoenberg, Magnetic Oscillations in Metals (Cambridge University Press, Cambridge, 1984).

[48] Y. Xu, S.-T. Wang, and L.-M. Duan, Weyl Exceptional Rings in a Three-Dimensional Dissipative Cold Atomic Gas, Phys. Rev. Lett. 118, 045701 (2017).

[49] Y. Chen and H. Zhai, Hall conductance of a non-Hermitian Chern insulator, Phys. Rev. B 98, 245130 (2018).

[50] T. M. Philip, M. R. Hirsbrunner, and M. J. Gilbert, Loss of Hall conductivity quantization in a non-Hermitian quantum anomalous Hall insulator, Phys. Rev. B 98, 155430 (2018).

[51] M. R. Hirsbrunner, T. M. Philip, and M. J. Gilbert, Topology and observables of the non-Hermitian Chern insulator, Phys. Rev. B 100, 081104(R) (2019).

[52] C. Wang and X. R. Wang, Non-quantized edge channel conductance and zero conductance fluctuation in non-Hermitian Chern insulators, arXiv:1901.06982.

[53] J. Mitscherling, Longitudinal and anomalous Hall conductivity of a general two-band model, Phys. Rev. B 102, 165151 (2020).

[54] N. Silberstein, J. Behrends, M. Goldstein, and R. Ilan, Berry connection induced anomalous wave-packet dynamics in nonHermitian systems, Phys. Rev. B 102, 245147 (2020).

[55] S. Groenendijk, T. L. Schmidt, and T. Meng, Universal Hall conductance scaling in non-Hermitian Chern insulators, Phys. Rev. Research 3, 023001 (2021).

[56] J.-H. Wang, Y.-L. Tao, and Y. Xu, Anomalous transport induced by non-Hermitian anomalous Berry connection in nonHermitian systems, arXiv:2109.08155. 